ISSN 0258-7122 (Print), 2408-8293 (Online)

Bangladesh J. Agril. Res. 42(1): 121-136, March 2017

\title{
NUTRIENT BALANCE UNDER WHEAT-MUNGBEAN-T. AMAN CROPPING PATTERN IN CALCAREOUS SOILS OF BANGLADESH
}

\author{
M. A. QUDDUS ${ }^{1}$, J. ABEDIN MIAN ${ }^{2}$, H. M. NASER ${ }^{3}$ \\ M. A. HOSSAIN ${ }^{4}$ AND A. K. M. SALAUDDIN ${ }^{5}$
}

\begin{abstract}
Field experiments on Wheat-Mungbean-T. aman cropping pattern were conducted at Regional Pulses Research Station (RPRS), Madaripur in calcareous soils under Low Ganges River Floodplain (AEZ-12) during 2009 to 2010 to prepare nutrient balance sheet for the cropping pattern and fertilizer recommendation for study area. The treatments were $\mathrm{T}_{1}=$ Control; $\mathrm{T}_{2}=$ Farmer's practice; $\mathrm{T}_{3}=$ AEZ based recommended fertilizer dose and $\mathrm{T}_{4}=$ Soil test based fertilizer dose. The experiment was laid out in RCBD with three replications. Results revealed that the average yields of wheat, mungbean and T. aman ranged from 1517 to $3124 \mathrm{~kg} \mathrm{ha}^{-1}, 1320$ to $1863 \mathrm{~kg} \mathrm{ha}^{-1}$ and 2974 to $4859 \mathrm{~kg} \mathrm{ha}^{-1}$, respectively. Grain yield of all crops increased significantly higher in soil test based (STB) fertilizer treatment $\left(\mathrm{T}_{4}\right)$ over the other treatments. Among the major nutrients, the magnitude of negative balance was greater with $\mathrm{N}$ and $\mathrm{K}$ followed by $\mathrm{Mg}$ and $\mathrm{Ca}$. The negative balance of $\mathrm{N}\left(-56.0\right.$ to $\left.-183 \mathrm{~kg} \mathrm{ha}^{-1}\right), \mathrm{K}$ (71.0 to $\left.-167 \mathrm{~kg} \mathrm{ha}^{-1}\right), \mathrm{Ca}\left(-7.50\right.$ to $\left.-27.1 \mathrm{~kg} \mathrm{ha}^{-1}\right)$, and $\mathrm{Mg}\left(-16.7\right.$ to $\left.-35.7 \mathrm{~kg} \mathrm{ha}^{-1}\right)$ was observed in all the managements might be due to added lower amount of nutrients in soil and higher removal by the crops from the soil. Positive balance of $\mathrm{P}$ indicated that the added amount of $\mathrm{P}$ is larger than the removal; $\mathrm{P}$ fertilization was enough to make apparent balances positive. Across various treatments, there was some amount of positive apparent $\mathrm{S}$ balance except absolute control plots and farmer's practice. On the other hand, $\mathrm{Zn}$ and $\mathrm{B}$ balance in the system was neutral to slightly positive. Results revealed that, $\mathrm{N}$, $\mathrm{K}$, $\mathrm{Ca}$, and $\mathrm{Mg}$ balance after two years of cropping was negative regardless of soil type and management strategies adopted. There was an improvement in organic matter in all treatments where biomasses of mungbean were incorporated. Organic matter, N, P, S, Zn and B status in soil was improved due to soil test based fertilization over the initial status. Considering the gross margin and soil fertility the soil test based (STB) fertilizer management practice is economically profitable and sustainable.
\end{abstract}

Keywords: Cropping pattern, management, nutrient balance, calcareous soils.

\section{Introduction}

Cropping pattern means yearly sequence of crop production followed in an area. The pattern in an area depends largely on agro-climatic, technical and

${ }^{1}$ Senior Scientific Officer, Horticulture Research Centre, Bangladesh Agricultural Research Institute (BARI), Gazipur, ${ }^{4}$ Principal Scientific Officer, PRSS, BARI, Gazipur, ${ }^{3}$ Principal Scientific Officer, Soil Science Division, BARI, Gazipur, ${ }^{2}$ Professor, Department of Soil Science, BAU, Mymensingh, ${ }^{5}$ Consultant, FAO, Dhaka, Bangladesh. 
institutional factors. Among food grain crops, rice occupies the first position both in terms of areas and production, next to wheat (Ghosh, 2011). These cereal based patterns cause considerable depletion of soil fertility and threat to long-term crop productivity. Besides that, farmers of Bangladesh use imbalanced of fertilizers for crop production leading to degraded in soil health and fertility (Ali et al., 2010). High yielding varieties of crops uptake higher amount of nutrient from soils resulting dwindling soil organic matter and deterioration of native soil fertility, poses a serious threat to long-term sustainability of crop production. Moreover, continuous cropping without adequate replacement of removed nutrients in harvested materials, nutrient loss through erosion, leaching and gaseous emission deplete soil fertility and causes soil organic matter level to decline ( $\mathrm{Yu}$ et al., 2014). The bulk of the literature indicates that, apart from residue management, cropping system productivity may become sustainable through integrated use of organic and inorganic nutrients.

Hence, it is important to develop a cropping system based fertilizer dose for specific agro-ecological zones. Low levels of plant nutrients (macro and micro) in many soils especially calcareous soil accompanied with improper nutrient management are major constraints for food security and malnutrition. Quantification of the loss or gain of nutrients under different cropping patterns has not been done properly. Nutrient balance is an important tool for assessing the fate of native and added nutrients in soils. Plant fertilization and yield increase is the main objectives of nutrient balance calculations (FRG, 2012).

Therefore, the existing situations are appeared to be threatening to the whole agricultural system. In this situation, introducing legume crop and proper fertility management practices in the existing cropping patterns is the only means of rejuvenation of soil as well as increase in total national product. Considering the above circumstances, the study was under taken to prepare nutrient balance sheet for Wheat-Mungbean-T. aman cropping pattern and fertilizer recommendation for the study area.

\section{Materials and method}

The field experiments were conducted for consecutive two years from rabi 2008-09 to October 2010 at Regional Pulses Research Station (RPRS), BARI, Madaripur ( $23^{\circ} 10^{\prime} 53^{\prime \prime} \mathrm{N}$ latitude and $90^{\circ} 11^{\prime} 28^{\prime \prime} \mathrm{E}$ longitude) at an elevation of $7.0 \mathrm{~m}$ above the sea level. The land belongs to the agro ecological zone, Low Ganges River Floodplain (AEZ-12) and Gopalpur soil series (Soil taxonomy: Aquic Eutrochrepts). The soil had neutral pH (7.3) and loamy in texture. The other soil properties were $1.32 \%$ organic matter, total $\mathrm{N}$ was $0.063 \%$, exchangeable $\mathrm{K}, \mathrm{Ca}$ and $\mathrm{Mg}$ were 0.14 meq. $100 \mathrm{~g}^{-1}, 10.3$ meq. $100 \mathrm{~g}^{-1}$ and 3.10 meq. $100 \mathrm{~g}^{-1}$, respectively. The available $\mathrm{P}, \mathrm{S}, \mathrm{Zn}$ and B were $13.5 \mu \mathrm{g} \mathrm{g}^{-1}, 18 \mu \mathrm{g}$ $\mathrm{g}^{-1}, 1.20 \mu \mathrm{g} \mathrm{g}^{-1}$ and $0.14 \mu \mathrm{g} \mathrm{g}^{-1}$, respectively. Cropping system Wheat-Mungbean- 
T. aman was considered for the study. The experiments were carried out over the three crop seasons such as Rabi, Kharif-I and Kharif-II. Wheat, mungbean and T. aman were grown in rabi, Kharif-I and Kharif-II season, respectively. There were four treatments along with control for each crop. The treatments were $\mathrm{T}_{1}=$ Control; $\mathrm{T}_{2}=$ Farmer's practice; $\mathrm{T}_{3}=$ AEZ based recommended fertilizer dose and $\mathrm{T}_{4}=$ Soil test based fertilizer dose. The descriptions of treatments are given in Table 1.

Table 1. Rates of fertilizers $\left(\mathrm{kg} \mathrm{ha}^{-1}\right)$ for wheat, mungbean and T. aman

\begin{tabular}{cll|l}
\hline Treatments & \multicolumn{1}{c|}{ Wheat } & \multicolumn{1}{c}{ Mungbean } & \multicolumn{1}{c}{ T. aman } \\
\hline $\mathrm{T}_{1}$ & Control & Control & Control \\
$\mathrm{T}_{2}$ & $\mathrm{~N}_{85} \mathrm{P}_{24} \mathrm{~K}_{24}$ & $\mathrm{~N}_{23} \mathrm{P}_{15} \mathrm{~K}_{8}$ & $\mathrm{~N}_{70} \mathrm{P}_{10} \mathrm{~K}_{15}$ \\
$\mathrm{~T}_{3}$ & $\mathrm{~N}_{90} \mathrm{P}_{16} \mathrm{~K}_{25} \mathrm{~S}_{8} Z_{1} \mathrm{~B}_{0.5}$ & $\mathrm{~N}_{15} \mathrm{P}_{18} \mathrm{~K}_{9} \mathrm{~S}_{8}$ & $\mathrm{~N}_{66} \mathrm{P}_{6} \mathrm{~K}_{12} \mathrm{~S}_{7} Z_{1}$ \\
$\mathrm{~T}_{4}$ & $\mathrm{~N}_{120} \mathrm{P}_{22} \mathrm{~K}_{62} \mathrm{~S}_{25} \mathrm{Zn}_{2} \mathrm{~B}_{1}$ & $\mathrm{~N}_{21} \mathrm{P}_{23} \mathrm{~K}_{30} \mathrm{~S}_{18} Z_{2} \mathrm{~B}_{1.5}$ & $\mathrm{~N}_{100} \mathrm{P}_{14} \mathrm{~K}_{66} \mathrm{~S}_{6} \mathrm{Zn}_{1.5} \mathrm{~B}_{1}$ \\
\hline
\end{tabular}

$\mathrm{T}_{1}=$ Control, $\mathrm{T}_{2}=$ Farmers' practice, $\mathrm{T}_{3}=$ AEZ based recommended fertilizer dose, $\mathrm{T}_{4}=$ Soil test based fertilizer dose.

The experiment was laid out in Randomized Complete Block Design (RCBD) with three replications. The unit plot size was $4 \mathrm{~m} \times 3 \mathrm{~m}$ for all crops having the spacing of $30 \mathrm{~cm} \times 10 \mathrm{~cm}$ for wheat, $40 \mathrm{~cm} \times 10 \mathrm{~cm}$ for mungbean and $20 \mathrm{~cm} \times$ $15 \mathrm{~cm}$ for T. aman rice. The layout was kept undisturbed for the cropping system over two years. The amount of all fertilizers, except urea in rice crop of each treatment was applied to respective plot at the time of final land preparation. Fertilizers were mixed with soil by spading. Urea was applied in three equal splits for T. aman rice (first split was applied immediately after seedling establishment, the second split during maximum tillering stage and before panicle initiation stage last split was applied). The sources of N, P, K, S, Zn and B were urea, triple super phosphate, muriate of potash, gypsum, zinc sulphate and boric acid, respectively. Wheat (var. BARI Gam-24) seeds were sown on 22 November 2008 and 23 November 2009. Mungbean (BARI Mung-6) seeds were sown on 18 March 2009 and on 27 March 2010. T. aman rice (var. BRRI dhan33) seedlings (30 days old) were transplanted on 23 July 2009 and on 9 July 2010. The crop wise seed rate was-wheat: $120 \mathrm{~kg} \mathrm{ha}^{-1}$, mungbean: $30 \mathrm{~kg} \mathrm{ha}^{-1}$ and T. aman rice: $25 \mathrm{~kg} \mathrm{ha}^{-1}$. Intercultural operations like irrigation, weeding and plant protection measures (insecticides and fungicides) were done as and when required. The transplanted rice seedlings were nursed properly in the seedbed. The crops were harvested after maturity. Data on yield and yield contributing characters of all crops from all plots were recorded. The yields data were expressed as $\mathrm{kg} \mathrm{ha}^{-1}$ which was adjusted to $14 \%$ moisture content for $\mathrm{T}$. aman rice and $12 \%$ moisture for wheat and mungbean. Analysis of variance (ANOVA) for the yield and yield contributing characters and different nutrient content was done following the principle of F-statistics and the mean values were separated by Duncan's Multiple Range Test (DMRT) using MSTAT-C software. 
Soil solutions were collected at intervals of 15 days starting from the date after transplantation with the help of $50 \mathrm{ml}$ plastic syringe and analyzed for determined nutrient leaching loss. Soil solution was collected at intervals of 15 days starting from the date after transplantation to harvest of rice crop with the help of $50 \mathrm{ml}$ plastic syringe. The samples were brought to the laboratory immediately after collection, filtered through Whatman No. 42 filter paper and preserved for the determination of $\mathrm{N}, \mathrm{P}, \mathrm{K}, \mathrm{S}, \mathrm{Ca}, \mathrm{Mg}, \mathrm{Zn}$ and $\mathrm{B}$. Rain and irrigation water were collected and analyzed for determining the nutrients $(\mathrm{N}, \mathrm{P}$, $\mathrm{K}, \mathrm{S}, \mathrm{Ca}, \mathrm{Mg}, \mathrm{Zn}$ and $\mathrm{B}$ ) added to the soil. In calculating percolation water ( $\mathrm{L} \mathrm{m}^{-}$ ${ }^{2}$ ) the formula $\mathrm{Q}=-\mathrm{K}_{\mathrm{w}} \mathrm{AT} \cdot \Delta \Psi_{\mathrm{h}} / \Delta \mathrm{z}$ given by Hanks and Ashcroft (1980) was used. Where, $Q=$ Quantity of water $K_{w}=$ Hydraulic conductivity, $A=$ Area, $T=$ Time, $\mathrm{H}=$ Difference in hydraulic potential and $\mathrm{Z}=$ Difference between two points taking 0 to downward as negative. The hydraulic potential was again calculated by adding the component potentials as $\Psi_{\mathrm{h}}=\Psi_{\mathrm{m}}+\Psi_{\mathrm{p}}+\Psi_{\mathrm{z}}$ where $\mathrm{h}, \mathrm{m}, \mathrm{p}$, and $\mathrm{z}$ represent hydraulic, metric, pressure and gravitational potentials. Negative Q was considered as downward movement of water.

An apparent nutrient balance was made by considering the nutrient input and output. The inputs were $\mathrm{N}, \mathrm{P}, \mathrm{K}, \mathrm{S}, \mathrm{Ca}, \mathrm{Mg}, \mathrm{Zn}$ and $\mathrm{B}$ which were supplied from (i) fertilizer (ii) rainfall and (iii) irrigation water. On the other hand, the outputs were calculated from the (i) crop uptake and (ii) leaching loss by percolation in a cycle.

A partial budget (average of two years) was calculated by a standard procedure. Benefit cost ratio (BCR) was used as a tool of partial budget analysis. It is the ratio of gross return and total cost. Gross returns were calculated by multiplying yield with the price of output. Total costs were calculated by variable cost. Variable cost consisting market price of fertilizer, pesticides and labor wages for land preparation, ploughing, weeding, seed sowing and fertilizers application. Land used cost or rental value of land was not considered here. Cost and return were estimated of crops due to different treatment.

\section{Results and Discussion}

\section{Crops yields}

The grain and straw/stover yields of wheat and mungbean exhibited significant variation due to different nutrient management practices in the consecutive two years (Table 2). Grain yield of wheat and mungbean increased significantly being higher under soil test based (STB) fertilizer treatment $\left(\mathrm{T}_{4}\right)$ over the other treatments. This indicated that the treatment $\mathrm{T}_{4}$ was more balanced than that of $\mathrm{T}_{2}$ and $\mathrm{T}_{3}$. Balanced fertilization through soil test based treatment produce higher 
yields of crops as well as sustains soil fertilty (Hossain et al., 2008). Biswas et al. (2009) found that balanced fertilizer management practice $\left(\mathrm{N}_{20} \mathrm{P}_{20} \mathrm{~K}_{20} \mathrm{~S}_{10} \mathrm{Zn}_{2}\right)$ showed higher seed yield $\left(1214 \mathrm{~kg} \mathrm{ha}^{-1}\right)$. The average grain yields of wheat and mungbean ranged from 1517 to $3124 \mathrm{~kg} \mathrm{ha}^{-1}$ and 1320 to $1863 \mathrm{~kg} \mathrm{ha}^{-1}$, respectively. In case of straw/stover yield, the effects of treatments were statistically differed with some exception and significantly highest value found in $\mathrm{T}_{4}$ treatment. The highest seed and stover yields of mungbean due to soil test based balanced fertilization was also found by other researchers (Kumar and Singh 2009). The lowest grain and straw/stover yields of wheat and mungbean were found in control $\mathrm{T}_{1}$ treatment in both the years. The percent grain yield of wheat and mungbean increased over control due to different nutrient management practices were 43 to $106 \%$ and 15 to $41 \%$ for calcareous soils (Madaripur), respectively. Most of the yield contributing characters of wheat and mungbean were highly responded to soil test based fertilization $\left(\mathrm{T}_{4}\right)$ followed by AEZ based fertilization $\left(\mathrm{T}_{3}\right)$. Aggarwal et al. (1997) also found that incorporation of green manure and chemicl fertilizer into soil which enhanced the yield contributing characters of wheat. Ved Ram et al. (2008) also observed that the application of N, P, K, S and Zn nutrients favoured the seeds per pod and 1000 seed weight.

The grain and straw yields of T. aman ( $3^{\text {rd }}$ crop) responded significantly to different nutrient management practices in both the years at calcareous soils (Table 2). The grain yield recorded from the treatment soil test based fertilizer dose and BARC recommended fertilizer dose $\left(\mathrm{T}_{3}\right)$ was statistically identical during 2009 and higher than farmer's practice $\left(\mathrm{T}_{2}\right)$ and control treatment. In case of straw yield, the treatments soil test based fertilizer dose $\left(\mathrm{T}_{4}\right)$ and BARC recommended fertilizer dose $\left(\mathrm{T}_{3}\right)$ differed significantly in 2010 but in 2009 they were statistically alike although soil test based fertilizer dose dominated over $\mathrm{T}_{3}$. Rahman et al. (2011) observed that the grain and straw yields of $\mathrm{T}$. aman were favoured by balanced nutrient application. Similar results were also observed by Biswas et al. (2009). The yield of T. aman was comparatively higher in first year than in second year in all treatments except control. The windy weather along with heavy shower at the flowering and ripening stage hampered pollination as a consequence the yield declined in second year. The lowest grain and straw yields were found in the control treatment. The grain yield ( 2 years' average) of T. aman varied from 2974 to $4859 \mathrm{~kg} \mathrm{ha}^{-1}$. The different nutrient management practices produced 32 to $63 \%$ yield increased over the control. Islam et al. (1996) also reported $42 \%$ yield increase of rice over control due to balanced fertilization. 
Table 2. Effect of nutrient management practices on grain and straw/stover yields of Wheat-Mungbean-T.aman cropping sequence

\begin{tabular}{|c|c|c|c|c|c|c|c|}
\hline \multirow{3}{*}{ Treatment } & \multicolumn{4}{|c|}{ Grain yield $\left(\mathrm{kg} \mathrm{ha}^{-1}\right)$} & \multicolumn{3}{|c|}{ Straw/stover yield $\left(\mathrm{kg} \mathrm{ha}^{-1}\right)$} \\
\hline & 2009 & 2010 & mean & $\begin{array}{c}\% \text { of } \\
\text { increase } \\
\text { over } \\
\text { control }\end{array}$ & 2009 & 2010 & mean \\
\hline & \multicolumn{7}{|c|}{ Wheat } \\
\hline Control $\left(\mathrm{T}_{1}\right)$ & $1555 d$ & $1478 d$ & 1517 & - & $2302 d$ & $2189 d$ & 2245 \\
\hline F. practice $\left(\mathrm{T}_{2}\right)$ & $2061 c$ & $2168 c$ & 2168 & 43 & $2499 \mathrm{c}$ & $2712 \mathrm{c}$ & 2605 \\
\hline $\mathrm{AEZ}\left(\mathrm{T}_{3}\right)$ & $2804 b$ & $2933 b$ & 2868 & 89 & $3471 b$ & $3546 b$ & 3508 \\
\hline $\mathrm{STB}\left(\mathrm{T}_{4}\right)$ & $3019 a$ & $3229 a$ & 3124 & 106 & $3599 a$ & $3767 a$ & 3683 \\
\hline CV $(\%)$ & 2.52 & 2.88 & - & - & 3.16 & 3.46 & - \\
\hline \multirow[t]{2}{*}{$\mathrm{LSD}_{0.05}$} & 161.85 & 172.5 & - & - & 246.3 & 259.1 & - \\
\hline & \multicolumn{7}{|c|}{ Mungbean } \\
\hline Control $\left(\mathrm{T}_{1}\right)$ & 1360d & $1280 \mathrm{~d}$ & 1320 & - & $2613 c$ & $2590 \mathrm{~d}$ & 2602 \\
\hline $\begin{array}{l}\text { F. practice } \\
\left(\mathrm{T}_{2}\right)\end{array}$ & $1566 c$ & $1467 \mathrm{c}$ & 1517 & 15 & $2912 b$ & $2844 c$ & 2878 \\
\hline $\operatorname{AEZ}\left(\mathrm{T}_{3}\right)$ & $1701 b$ & $1620 b$ & 1661 & 26 & $3056 b$ & $3004 b$ & 2950 \\
\hline $\mathrm{STB}\left(\mathrm{T}_{4}\right)$ & $1926 a$ & $1800 \mathrm{a}$ & 1863 & 41 & $3110 \mathrm{a}$ & $3075 a$ & 3093 \\
\hline $\mathrm{CV}(\%)$ & 2.32 & 3.20 & - & - & 3.31 & 4.33 & - \\
\hline \multirow[t]{2}{*}{$\mathrm{LSD}_{0.05}$} & 162 & 240 & - & - & 175 & 296 & - \\
\hline & \multicolumn{7}{|c|}{ T. aman } \\
\hline Control $\left(\mathrm{T}_{1}\right)$ & $3211 \mathrm{c}$ & $2736 d$ & 2974 & - & $3376 c$ & $2870 \mathrm{~d}$ & 3123 \\
\hline $\begin{array}{l}\mathrm{F} . \quad \text { practice } \\
\left(\mathrm{T}_{2}\right)\end{array}$ & $3973 b c$ & $3859 \mathrm{c}$ & 3916 & 32 & $4128 b c$ & $3958 \mathrm{c}$ & 4043 \\
\hline $\operatorname{AEZ}\left(\mathrm{T}_{3}\right)$ & $4518 \mathrm{ab}$ & $4500 \mathrm{~b}$ & 4509 & 52 & $4614 a b$ & $4590 \mathrm{~b}$ & 4602 \\
\hline $\mathrm{STB}\left(\mathrm{T}_{4}\right)$ & $4938 \mathrm{a}$ & $4779 \mathrm{a}$ & 4859 & 63 & $5036 a$ & $4909 a$ & 4972 \\
\hline CV (\%) & 5.21 & 3.80 & - & - & 4.94 & 3.74 & - \\
\hline $\mathrm{LSD}_{0.05}$ & 502.5 & 318.8 & - & - & 446.7 & 321.7 & - \\
\hline
\end{tabular}

Values within the same column with a common letter do not differ significantly $(\mathrm{p}=0.05)$. $\mathrm{F}=$ Farmers.

\section{Nutrient uptake}

Nutrient management practices have made significant effect to uptake of $\mathrm{N}, \mathrm{P}, \mathrm{K}$, $\mathrm{S}, \mathrm{Ca}, \mathrm{Mg}, \mathrm{Zn}$ and B by wheat, mungbean and T. aman under Wheat-MungbeanT. aman cropping pattern during $2009 \& 2010$ (Tables $3 \& 4$ ). The soil test based fertilizer treatment $\left(\mathrm{T}_{4}\right)$ showed the significantly higher nutrients uptake by wheat 
in both the years. Similar results were found by Jahan et al. (2015a). The second highest uptake was observed in $T_{3}$ which was followed by $T_{2}$. The nutrient uptake followed the order: $\mathrm{N}>\mathrm{K}>\mathrm{Ca}>\mathrm{Mg}>\mathrm{P}>\mathrm{S}>\mathrm{Zn}>\mathrm{B}$. Almost all nutrient found higher uptakes by mungbean in soil test based fertilizer treatment $\left(\mathrm{T}_{4}\right)$ followed by $T_{3}$ and then $T_{2}$ treatments. The soil test based fertilizer treatment $\left(T_{4}\right)$ influenced to uptake highest amount of all nutrients by T. aman rice followed by $\mathrm{T}_{3}$ and than $\mathrm{T}_{2}$ treatment. The lower nutrient uptake was found in control $\left(\mathrm{T}_{1}\right)$ treatment by all crops (Tables $3 \& 4$ ). The total uptake of nutrients by crops (wheat+mungbean+T. aman) ranged from $183-305 \mathrm{~kg} \mathrm{~N} \mathrm{ha}^{-1}, 21.1-38.5 \mathrm{~kg} \mathrm{P}$ ha ${ }^{1}, 148-223 \mathrm{~kg} \mathrm{~K} \mathrm{ha}^{-1}, 9.45-18.6 \mathrm{~kg} \mathrm{~S}^{-1}, 37.8-60.5 \mathrm{~kg} \mathrm{Ca} \mathrm{ha}^{-1}, 28.4-46.6 \mathrm{~kg} \mathrm{Mg}$ $\mathrm{ha}^{-1}, 0.47-0.82 \mathrm{~kg} \mathrm{Zn} \mathrm{ha}^{-1}$ and $0.25-0.43 \mathrm{~kg} \mathrm{~B} \mathrm{ha}^{-1}$. These observations are in agreement with Tarafder et al. (2008) in potato-boro-T. aman rice cropping pattern. The uptake of all nutrients due to different nutrients management practices followed almost same trend (Fig. $1 \& 2$ ).

Table 3. Effect of nutrient management practices on nutrient uptake $\left(\mathrm{kg} \mathrm{ha}^{-1}\right)$ by Wheat-Mungbean-T. aman (grain+straw/stover) cropping pattern

\begin{tabular}{|c|c|c|c|c|c|c|c|c|}
\hline \multirow{3}{*}{ Treatment } & \multicolumn{2}{|c|}{$\mathrm{N}$} & \multicolumn{2}{|c|}{$\mathrm{P}$} & \multicolumn{2}{|c|}{$\mathrm{K}$} & \multicolumn{2}{|c|}{$S$} \\
\hline & 2009 & 2010 & 2009 & 2010 & 2009 & 2010 & 2009 & 2010 \\
\hline & \multicolumn{8}{|c|}{ Wheat } \\
\hline Control $\left(\mathrm{T}_{1}\right)$ & $42.2 \mathrm{~d}$ & $39.3 d$ & $6.00 \mathrm{c}$ & $5.63 c$ & $35.7 d$ & $33.4 d$ & $3.17 \mathrm{~d}$ & $2.64 b$ \\
\hline F. practice $\left(T_{2}\right)$ & $54.0 \mathrm{c}$ & $56.7 \mathrm{c}$ & $8.16 b$ & $8.35 b$ & $40.2 c$ & $42.6 c$ & $4.06 \mathrm{c}$ & $3.85 b$ \\
\hline $\operatorname{AEZ}\left(\mathrm{T}_{3}\right)$ & $74.7 \mathrm{~b}$ & $76.7 b$ & $11.2 \mathrm{a}$ & $11.7 \mathrm{a}$ & $56.2 \mathrm{~b}$ & $57.1 \mathrm{~b}$ & $5.86 \mathrm{~b}$ & $5.42 \mathrm{a}$ \\
\hline $\operatorname{STB}\left(\mathrm{T}_{4}\right)$ & $81.6 \mathrm{a}$ & $85.8 \mathrm{a}$ & $12.5 \mathrm{a}$ & $13.2 \mathrm{a}$ & $59.8 \mathrm{a}$ & $61.8 \mathrm{a}$ & $6.56 \mathrm{a}$ & $6.24 \mathrm{a}$ \\
\hline $\mathrm{CV}(\%)$ & 2.24 & 3.57 & 8.63 & 10.25 & 2.72 & 3.62 & 6.10 & 8.18 \\
\hline \multirow[t]{2}{*}{$\mathrm{LSD}_{0.05}$} & 3.12 & 4.61 & 1.63 & 1.99 & 2.65 & 3.52 & 1.19 & 1.29 \\
\hline & \multicolumn{8}{|c|}{ Mungbean } \\
\hline Control $\left(\mathrm{T}_{1}\right)$ & $79.2 d$ & $75.3 d$ & $8.66 \mathrm{~d}$ & $7.98 \mathrm{~d}$ & $74.7 d$ & $70.1 d$ & $3.19 \mathrm{c}$ & $2.71 \mathrm{~b}$ \\
\hline F.practice $\left(\mathrm{T}_{2}\right)$ & $91.1 \mathrm{c}$ & $86.2 \mathrm{c}$ & $9.96 c$ & $9.10 \mathrm{c}$ & $84.6 c$ & $80.9 c$ & $3.60 \mathrm{~b}$ & $3.03 \mathrm{~b}$ \\
\hline $\operatorname{AEZ}\left(\mathrm{T}_{3}\right)$ & $98.6 b$ & $94.6 b$ & $10.9 \mathrm{~b}$ & $10.3 b$ & $89.8 b$ & $87.1 \mathrm{~b}$ & $4.32 \mathrm{~b}$ & $3.72 \mathrm{ab}$ \\
\hline $\operatorname{STB}\left(\mathrm{T}_{4}\right)$ & $108 \mathrm{a}$ & $103 a$ & $12.3 \mathrm{a}$ & $11.5 \mathrm{a}$ & $95.0 \mathrm{a}$ & $91.9 \mathrm{a}$ & $5.11 \mathrm{a}$ & $4.44 \mathrm{a}$ \\
\hline $\mathrm{CV}(\%)$ & 2.45 & 1.85 & 4.57 & 5.16 & 1.21 & 1.29 & 8.01 & 9.25 \\
\hline \multirow[t]{2}{*}{$\mathrm{LSD}_{0.05}$} & 3.10 & 1.53 & 1.14 & 1.03 & 1.95 & 1.63 & 1.23 & 1.00 \\
\hline & \multicolumn{8}{|c|}{ T. aman } \\
\hline Control $\left(\mathrm{T}_{1}\right)$ & $72.2 d$ & $57.8 d$ & $7.85 \mathrm{~d}$ & $6.13 d$ & $44.8 \mathrm{~d}$ & $37.3 d$ & $4.31 \mathrm{c}$ & $2.82 \mathrm{c}$ \\
\hline F.practice $\left(\mathrm{T}_{2}\right)$ & $91.8 \mathrm{c}$ & $87.6 c$ & $10.5 \mathrm{c}$ & $8.95 \mathrm{c}$ & $55.8 \mathrm{c}$ & $53.2 \mathrm{c}$ & $5.69 \mathrm{~b}$ & $4.70 \mathrm{~b}$ \\
\hline $\operatorname{AEZ}\left(\mathrm{T}_{3}\right)$ & $106 \mathrm{~b}$ & $104 b$ & $12.3 b$ & $11.3 \mathrm{~b}$ & $62.5 b$ & $62.2 \mathrm{~b}$ & $6.86 a$ & $6.36 \mathrm{ab}$ \\
\hline $\mathrm{STB}\left(\mathrm{T}_{4}\right)$ & $118 \mathrm{a}$ & $113 a$ & $14.4 \mathrm{a}$ & $13.0 \mathrm{a}$ & $69.3 \mathrm{a}$ & $67.5 \mathrm{a}$ & $7.99 a$ & $6.79 a$ \\
\hline $\mathrm{CV}(\%)$ & 1.39 & 2.25 & 4.46 & 4.86 & 2.05 & 2.47 & 5.29 & 4.46 \\
\hline $\mathrm{LSD}_{0.05}$ & 2.70 & 3.68 & 1.01 & 1.45 & 2.38 & 2.49 & 1.00 & 0.87 \\
\hline
\end{tabular}

Values within the same column with a common letter do not differ significantly $(\mathrm{p}=0.05)$. $\mathrm{F}=$ Farmers. 
Table 4. Effect of nutrient management practices on nutrient uptake $\left(\mathrm{kg} \mathrm{ha}^{-1}\right)$ by Wheat-Mungbean-T. aman (grain+straw/stover) cropping pattern

\begin{tabular}{|c|c|c|c|c|c|c|c|c|}
\hline \multirow{3}{*}{ Treatment } & \multicolumn{2}{|c|}{$\mathrm{Ca}$} & \multicolumn{2}{|c|}{$\mathrm{Mg}$} & \multicolumn{2}{|c|}{$\mathrm{Zn}$} & \multicolumn{2}{|c|}{ B } \\
\hline & 2009 & 2010 & 2009 & 2010 & 2009 & 2010 & 2009 & 2010 \\
\hline & \multicolumn{8}{|c|}{ Wheat } \\
\hline Control $\mathrm{T}_{1}$ ) & $8.55 b$ & $7.55 d$ & $6.94 c$ & $6.08 b$ & $0.17 d$ & $0.16 \mathrm{~d}$ & $0.10 \mathrm{c}$ & $0.09 \mathrm{~d}$ \\
\hline F. practice $\left(T_{2}\right)$ & $10.2 b$ & $10.1 \mathrm{c}$ & $8.34 b$ & $7.97 \mathrm{~b}$ & $0.20 \mathrm{c}$ & $0.21 \mathrm{c}$ & $0.11 \mathrm{c}$ & $0.12 \mathrm{c}$ \\
\hline $\operatorname{AEZ}\left(\mathrm{T}_{3}\right)$ & $14.6 \mathrm{a}$ & $14.1 \mathrm{~b}$ & $11.8 \mathrm{a}$ & $11.2 \mathrm{~b}$ & $0.29 b$ & $0.31 b$ & $0.16 \mathrm{~b}$ & $0.17 b$ \\
\hline $\operatorname{STB}\left(\mathrm{T}_{4}\right)$ & $15.7 \mathrm{a}$ & $15.9 \mathrm{a}$ & $13.1 \mathrm{a}$ & $13.1 \mathrm{a}$ & $0.32 \mathrm{a}$ & $0.35 \mathrm{a}$ & $0.18 \mathrm{a}$ & $0.19 \mathrm{a}$ \\
\hline $\mathrm{CV}(\%)$ & 7.11 & 6.87 & 6.47 & 7.97 & 8.41 & 8.01 & 4.64 & 6.08 \\
\hline \multirow[t]{2}{*}{$\mathrm{LSD}_{0.05}$} & 1.73 & 1.64 & 1.29 & 1.81 & 0.02 & 0.02 & 0.04 & 0.019 \\
\hline & \multicolumn{8}{|c|}{ Mungbean } \\
\hline Contro $1\left(\mathrm{~T}_{1}\right)$ & $18.0 \mathrm{~d}$ & $17.2 \mathrm{~d}$ & $14.0 \mathrm{~d}$ & $12.9 \mathrm{~d}$ & $0.09 \mathrm{~b}$ & $0.08 \mathrm{~b}$ & $0.07 b$ & $0.06 \mathrm{~b}$ \\
\hline F.practice $\left(\mathrm{T}_{2}\right)$ & $20.2 c$ & $19.1 \mathrm{c}$ & $15.8 \mathrm{c}$ & $14.6 \mathrm{c}$ & $0.10 \mathrm{ab}$ & $0.09 \mathrm{ab}$ & $0.07 \mathrm{ab}$ & $0.07 \mathrm{ab}$ \\
\hline $\operatorname{AEZ~}\left(\mathrm{T}_{3}\right)$ & $21.9 b$ & $20.9 b$ & $17.2 \mathrm{~b}$ & $16.1 \mathrm{~b}$ & $0.11 \mathrm{ab}$ & $0.10 \mathrm{ab}$ & 0.09ab & $0.08 \mathrm{ab}$ \\
\hline $\mathrm{STB}\left(\mathrm{T}_{4}\right)$ & $23.6 \mathrm{a}$ & $22.4 \mathrm{a}$ & $18.6 \mathrm{a}$ & $17.4 \mathrm{a}$ & $0.12 \mathrm{a}$ & $0.11 \mathrm{a}$ & $0.10 \mathrm{a}$ & $0.09 \mathrm{a}$ \\
\hline CV (\%) & 4.05 & 3.81 & 4.53 & 3.77 & 6.23 & 5.26 & 7.02 & 6.76 \\
\hline \multirow[t]{2}{*}{$\mathrm{LSD}_{0.05}$} & 1.32 & 1.52 & 1.36 & 1.15 & 0.03 & 0.02 & 0.04 & 0.019 \\
\hline & \multicolumn{8}{|c|}{ T. aman } \\
\hline Control $\left(\mathrm{T}_{1}\right)$ & $13.6 \mathrm{~d}$ & $10.7 \mathrm{c}$ & $9.59 \mathrm{~d}$ & $7.31 \mathrm{~d}$ & $0.24 d$ & $0.20 \mathrm{~d}$ & $0.10 \mathrm{~d}$ & $0.09 \mathrm{~d}$ \\
\hline F.practice $\left(\mathrm{T}_{2}\right)$ & $17.1 \mathrm{c}$ & $15.7 b$ & $12.2 \mathrm{c}$ & $9.80 \mathrm{c}$ & $0.29 \mathrm{c}$ & $0.28 \mathrm{c}$ & $0.12 \mathrm{c}$ & $0.11 \mathrm{c}$ \\
\hline $\operatorname{AEZ}\left(\mathrm{T}_{3}\right)$ & $19.7 b$ & $18.7 \mathrm{ab}$ & $14.2 \mathrm{~b}$ & $12.8 \mathrm{~b}$ & $0.35 b$ & $0.34 b$ & $0.14 b$ & $0.15 b$ \\
\hline $\mathrm{STB}\left(\mathrm{T}_{4}\right)$ & $22.5 \mathrm{a}$ & $20.9 a$ & $16.5 \mathrm{a}$ & $14.6 \mathrm{a}$ & $0.37 \mathrm{a}$ & $0.36 a$ & $0.17 \mathrm{a}$ & $0.16 a$ \\
\hline CV (\%) & 4.60 & 5.12 & 4.94 & 5.51 & 5.02 & 4.52 & 4.69 & 3.79 \\
\hline $\mathrm{LSD}_{0.05}$ & 1.57 & 1.87 & 1.29 & 1.47 & 0.019 & 0.05 & 0.019 & 0.02 \\
\hline
\end{tabular}

Values within the same column with a common letter do not differ significantly $(\mathrm{p}=0.05)$. $\mathrm{F}=$ Farmers.

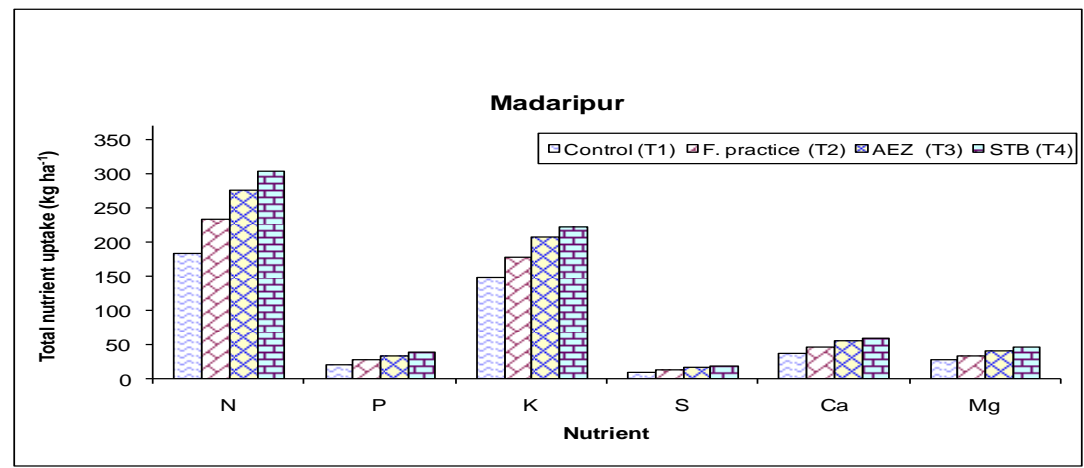

Fig. 1. Effect of fertilizer management practices on total uptake of nutrients by crops (wheat+mungbean+T. aman) under Wheat-Mungbean-T. aman cropping pattern at Madaripur. 


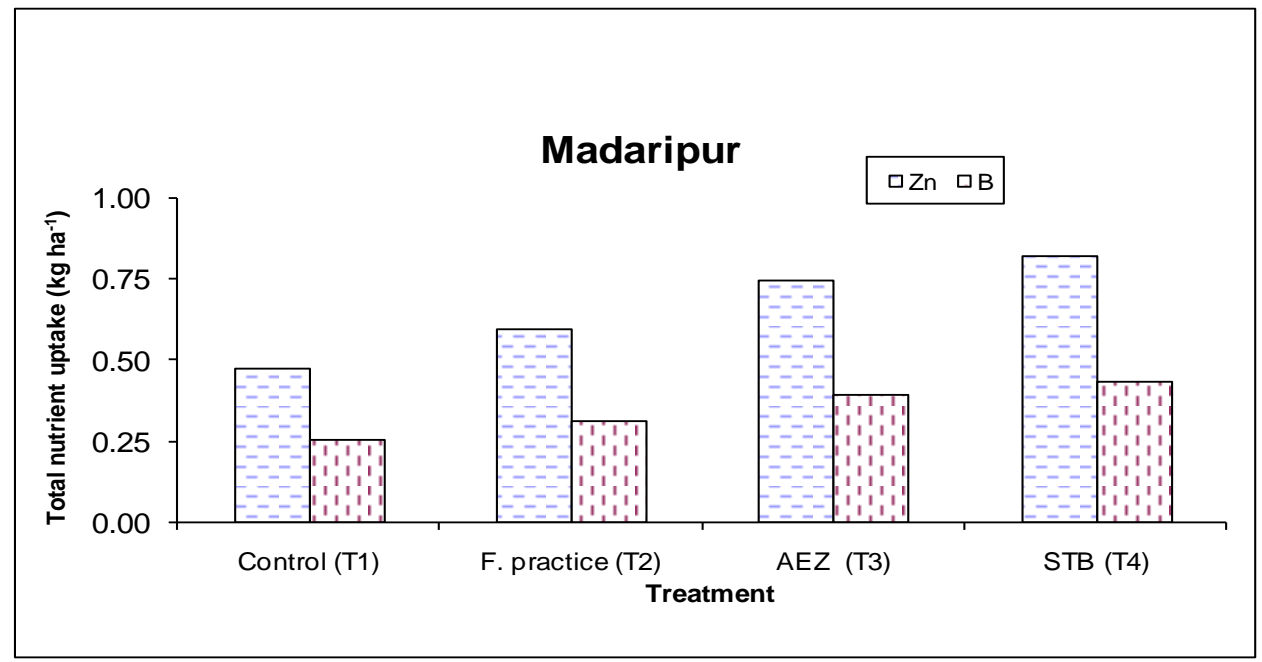

Fig. 2. Effect of fertilizer management practices on total uptake of zinc \& boron by crops (wheat+mungbean+T. aman) under Wheat-Mungbean-T. aman cropping pattern.

\section{Leaching of nutrients}

Loss through leaching was taken into account only to T. aman rice. We assumed that there would be no loss of nutrients through leaching from the soil during wheat and mungbean cultivation. Nutrient loss was calculated from the results of percolation water and nutrient concentration in soil solution. Nitrogen loss was ignored due to very low concentration in soil solution. Different nutrient management practices favoured the loss of $\mathrm{P}, \mathrm{K}, \mathrm{S}, \mathrm{Ca}, \mathrm{Mg}, \mathrm{Zn}$ and $\mathrm{B}$ element through leaching. The loss of nutrients (average of two years) through leaching ranged from 0.30 to $0.71 \mathrm{~kg} \mathrm{P} \mathrm{ha}^{-1}, 3.29$ to $11.9 \mathrm{~kg} \mathrm{~K} \mathrm{ha}^{-1}, 1.57$ to $4.35 \mathrm{~kg} \mathrm{~S} \mathrm{ha}^{-1}$, 15.3 to $20.5 \mathrm{~kg} \mathrm{Ca} \mathrm{ha}^{-1}, 9.66$ to $10.6 \mathrm{~kg} \mathrm{Mg} \mathrm{ha}^{-1}, 0.05$ to $0.12 \mathrm{~kg} \mathrm{Zn} \mathrm{ha}^{-1}$ and 0.10 to $0.37 \mathrm{~kg} \mathrm{~B} \mathrm{ha}^{-1}$. Katoh et al. (2003) reported that the amounts of nutrients leached by percolation ranged from $25-130 \mathrm{~kg} \mathrm{ha}^{-1} \mathrm{Ca}, 8-24 \mathrm{~kg} \mathrm{ha}^{-1} \mathrm{Mg}$, from -1 to $9 \mathrm{~kg} \mathrm{ha}^{-1} \mathrm{~K}$, respectively were lost each year from the soil layer during rice cultivation. The highest and lowest values of nutrients were always found in $\mathrm{T}_{4}$ and $\mathrm{T}_{1}$ treatments, respectively (Table 5).

Table 5. Effect of nutrient management practices on nutrient loss through leaching under Wheat-Mungbean-T. aman cropping pattern (average of two years)

\begin{tabular}{l|c|c|c|c|c|c|c}
\hline \multirow{2}{*}{ Treatment } & $\mathrm{P}$ & $\mathrm{K}$ & $\mathrm{S}$ & $\mathrm{Ca}$ & $\mathrm{Mg}$ & $\mathrm{Zn}$ & $\mathrm{B}$ \\
\cline { 2 - 8 } & \multicolumn{7}{c}{$\mathrm{kg} \mathrm{ha}^{-1}$} \\
\hline Control $\left(\mathrm{T}_{1}\right)$ & 0.30 & 3.29 & 1.57 & 15.3 & 9.66 & 0.05 & 0.10 \\
F. practice $\left(\mathrm{T}_{2}\right)$ & 0.60 & 8.60 & 2.49 & 18.1 & 10.4 & 0.06 & 0.10 \\
AEZ $\left(\mathrm{T}_{3}\right)$ & 0.66 & 11.1 & 3.93 & 18.9 & 10.5 & 0.11 & 0.35 \\
$\mathrm{STB}\left(\mathrm{T}_{4}\right)$ & 0.71 & 11.9 & 4.35 & 20.5 & 10.6 & 0.12 & 0.37 \\
\hline
\end{tabular}




\section{Total input of nutrients}

The nutrient input mainly from fertilizer but in this estimate, the nutrients supply from fertilizer, rainfall and irrigation under Wheat-Mungbean-T. aman cropping pattern. BNF was not considerd. Total input of nitrogen was $178-241 \mathrm{~kg} \mathrm{~N} \mathrm{ha}^{-1}$ of which the major part was added through fertilizer application, except in control treatment. Phosphorus input ranged from 0.55 to $59.5 \mathrm{~kg} \mathrm{ha}^{-1} \mathrm{yr}^{-1}$ and $\mathrm{K}$ from 5.75 to $164 \mathrm{~kg} \mathrm{ha}^{-1} \mathrm{yr}^{-1}$ (Table 6). The $\mathrm{S}$ input varied from 3.26 to $52.6 \mathrm{~kg} \mathrm{ha}^{-1} \mathrm{yr}^{-1}, \mathrm{Ca}$ and $\mathrm{Mg}$ input from 45.7 to $53.9 \mathrm{~kg} \mathrm{ha}^{-1} \mathrm{yr}^{-1}$ and 21.3 to $21.3 \mathrm{~kg} \mathrm{ha}^{-1} \mathrm{yr}^{-1}$, respectively. Input of $\mathrm{Zn}$ ranged from 0.076 to $5.58 \mathrm{~kg} \mathrm{ha}^{-1} \mathrm{yr}^{-1}$. Zinc and B input was estimated 0.076 to $5.58 \mathrm{~kg} \mathrm{ha}^{-1} \mathrm{yr}^{-1}$ and 0.34 to $3.84 \mathrm{~kg} \mathrm{ha}^{-1} \mathrm{yr}^{-1}$, respectively (Table 6).

Table 6. Total input of $\mathrm{N}, \mathrm{P}, \mathrm{K}, \mathrm{S}, \mathrm{Ca}, \mathrm{Mg}, \mathrm{Zn}$ and $\mathrm{B}$ from fertilizer, rainfall and irrigation under Wheat-Mungbean-T. aman cropping pattern

\begin{tabular}{l|c|c|c|c|c|c|c|c}
\hline \multirow{2}{*}{ Treatment } & $\mathrm{N}$ & $\mathrm{P}$ & $\mathrm{K}$ & $\mathrm{S}$ & $\mathrm{Ca}$ & $\mathrm{Mg}$ & $\mathrm{Zn}$ & $\mathrm{B}$ \\
\cline { 2 - 9 } & $2009-10$ & $2009-10$ & $2009-10$ & $2009-10$ & $2009-10$ & $2009-10$ & $2009-10$ & $2009-10$ \\
\cline { 2 - 9 } & 0.00 & 0.55 & 5.75 & 3.26 & 45.7 & 21.3 & 0.076 & 0.34 \\
Control( $\left(\mathrm{T}_{1}\right)$ & 178 & 49.5 & 52.8 & 3.41 & 51.7 & 21.3 & 0.076 & 0.34 \\
F. practice $\left(\mathrm{T}_{2}\right)$ & 171 & 40.5 & 51.8 & 26.5 & 51.3 & 21.3 & 2.076 & 0.84 \\
AEZ $\left(\mathrm{T}_{3}\right)$ & 241 & 59.5 & 164 & 52.6 & 53.9 & 21.3 & 5.58 & 3.84 \\
STB $\left(\mathrm{T}_{4}\right)$ & &
\end{tabular}

\section{Total output of nutrients}

Output of nitrogen with the harvested product was about double time larger than the sum of inputs. We could not include $\mathrm{N}$ from leaching. The output of phosphorus is also moderate (21.4 to $\left.39.2 \mathrm{~kg} \mathrm{P} \mathrm{ha}^{-1} \mathrm{yr}^{-1}\right)$. The removal of potassium is 2 to 4 folds greater than the sum of inputs. The removal of calcium with the yield and leaching is greater than the sum of inputs. For magnesium, the nutrient removal with the yield and leaching is about two times larger than the sum of inputs. The output of nutrients (mean of two years) ranged from 183 to $305 \mathrm{~kg} \mathrm{~N} \mathrm{ha}^{-1}, 21.4$ to $39.2 \mathrm{~kg} \mathrm{P} \mathrm{ha}^{-1}, 151$ to $235 \mathrm{~kg} \mathrm{~K} \mathrm{ha}^{-1}, 11.0$ to $22.9 \mathrm{~kg} \mathrm{~S}^{-1}$, 53.2 to $81.0 \mathrm{~kg} \mathrm{Ca} \mathrm{ha}^{-1}, 38.0$ to $57.0 \mathrm{~kg} \mathrm{Mg} \mathrm{ha}^{-1}, 0.52$ to $0.94 \mathrm{~kg} \mathrm{Zn} \mathrm{ha}^{-1}$ and 0.36 to $0.83 \mathrm{~kg} \mathrm{~B} \mathrm{ha}^{-1}$ (Table 7).

Table 7. Effect of nutrient management practices on total output (crop uptake and leaching loss) of nutrients by Wheat-Mungbean-T. aman cropping pattern (average of two years)

\begin{tabular}{|c|c|c|c|c|c|c|c|c|}
\hline \multirow{2}{*}{ Treatment } & $\mathrm{N}$ & $\mathrm{P}$ & $\mathrm{K}$ & $S$ & $\mathrm{Ca}$ & $\mathrm{Mg}$ & $\mathrm{Zn}$ & B \\
\hline & \multicolumn{8}{|c|}{ kg ha $^{-1}$} \\
\hline Control $\left(\mathrm{T}_{1}\right)$ & 183 & 21.4 & 151 & 11.0 & 53.2 & 38.0 & 0.52 & 0.36 \\
\hline F. practice $\left(T_{2}\right)$ & 234 & 28.1 & 188 & 15.0 & 64.0 & 45.0 & 0.65 & 0.40 \\
\hline $\operatorname{AEZ}\left(\mathrm{T}_{3}\right)$ & 277 & 35.0 & 219 & 20.2 & 74.0 & 52.0 & 0.85 & 0.75 \\
\hline $\mathrm{STB}\left(\mathrm{T}_{4}\right)$ & 305 & 39.2 & 235 & 22.9 & 81.0 & 57.0 & 0.94 & 0.83 \\
\hline
\end{tabular}




\section{Apparent nutrients balance}

An apparent nutrient balance was calculated considering the amount of added nutrient through fertilizer, rain, irrigation water minus the amount of nutrient removed by crops and losses occurred through crop harvest and leaching. However, the nutrient balance did not account for the addition of $\mathrm{N}$ from rainfall, irrigation water, or gaseous losses of $\mathrm{N}$ or BNF. Apparent balance of $\mathrm{N}, \mathrm{P}, \mathrm{K}, \mathrm{S}, \mathrm{Ca}, \mathrm{Mg}, \mathrm{Zn}$ and $\mathrm{B}$ are shown in Figs. $03 \&$ 04. The balance was mainly affected by different nutrient management practices. The apparent balance of $\mathrm{N}$ was negative in all the treatment and the soil depletion ranged from -56.0 to $-183 \mathrm{~kg} \mathrm{~N} \mathrm{ha}^{-1} \mathrm{yr}^{-1}$. Some researchers supported the results: in rice-maize system in Bangladesh, the apparent nutrient balances have been highly negative for $\mathrm{N}\left(-120\right.$ to $\left.-134 \mathrm{~kg} \mathrm{ha}^{-1} \mathrm{yr}^{-1}\right)$ (Timsina et al., 2010). In case of $\mathrm{P}$ balance which was negative in control treatment $\mathrm{T}_{1}$ and the $\mathrm{P}$ balance was positive in all the other treatment where $\mathrm{P}$ containing fertilizer was utilized. This evident indicated that $\mathrm{P}$ depletion was fewer amounts as compared added fertilizer. Saleque et al. (2006) expressed the same agreement.The balance of K was negative in all the treatments where the $\mathrm{K}$ mining ranged from -71.0 to $-167 \mathrm{~kg} \mathrm{~K} \mathrm{ha}^{-1} \mathrm{yr}^{-1}$. The maximum $\mathrm{K}$ depletion was observed in control plot and application of $\mathrm{K}$ gradually decreased the depletion with increasing rate. Bijay and Yadvinder (2002) observed that the depletion of $\mathrm{K}$ decreased with increasing rate of $\mathrm{K}$ application in soil. The negative $\mathrm{S}, \mathrm{Zn}$ and $\mathrm{B}$ balance was observed in control and farmers practice treatments ranged from -7.74 to -11.6 , -0.44 to -0.57 and -0.02 to $-0.06 \mathrm{~kg} \mathrm{ha}^{-1} \mathrm{yr}^{-1}$, respectively. Remaining treatments showed positive balance ranged from 6.30 to $29.7,1.23$ to 3.66 and 0.10 to $3.01 \mathrm{~kg} \mathrm{ha}^{-1} \mathrm{yr}^{-1}$, respectively. Among the treatments, the maximum positive balance was observed in STB followed by AEZ treatment. Alam et al. (2000) observed that $S$ balance was positive in soil due to integrated application of fertilizer and manure. The apparent balance for $\mathrm{Zn}$ and $\mathrm{B}$ was negative in the treatment of $T_{1}$ and $T_{2}$ and positive in $T_{3}$ and $T_{4}$ treatments due to their application. Similar results were also obtained by Hossain et al. (2008). Apparent balance for $\mathrm{Ca}$ and $\mathrm{Mg}$ was negative in all treatment. Calcium and $\mathrm{Mg}$ uptake was comparatively higher than that of supplied which got from soil, irrigation and rain water. The calcareous soil having more $\mathrm{CaCO}_{3}$, but the $\mathrm{Ca}$ balance was negative; it might be due to less soluble form in high $\mathrm{pH}$. Jensen and Thomas (2010) reported that soil $\mathrm{pH}$ greater than 7.2-7.5, phosphate ions tend to react quickly with $\mathrm{Ca}$ and $\mathrm{Mg}$ to form less soluble compounds. A long term study by Srinivasarao et al. (2014) on groundnut-fingermillet crop rotation, a negative balance to positive balance ( -315 to $12.37 \mathrm{~kg} \mathrm{Ca} \mathrm{ha}^{-1} \mathrm{yr}^{-1}$ ) was found for $\mathrm{Ca}$. On the other hand, $\mathrm{Mg}$ balance was found to be negative which ranged from -64 to $-207 \mathrm{Kg} \mathrm{Mg} \mathrm{ha-1} \mathrm{yr}^{-1}$. 


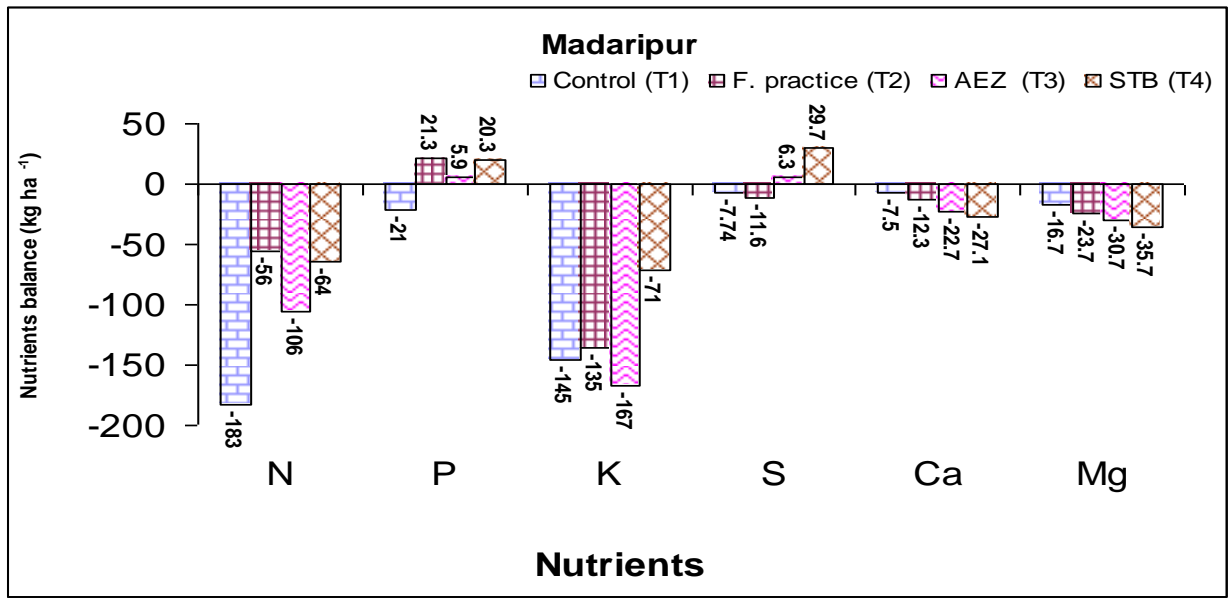

Fig.3. Apparent nutrient balance of N, P, K, S, Ca and Mg under Wheat-Mungbean -T. aman cropping pattern at Madaripur.

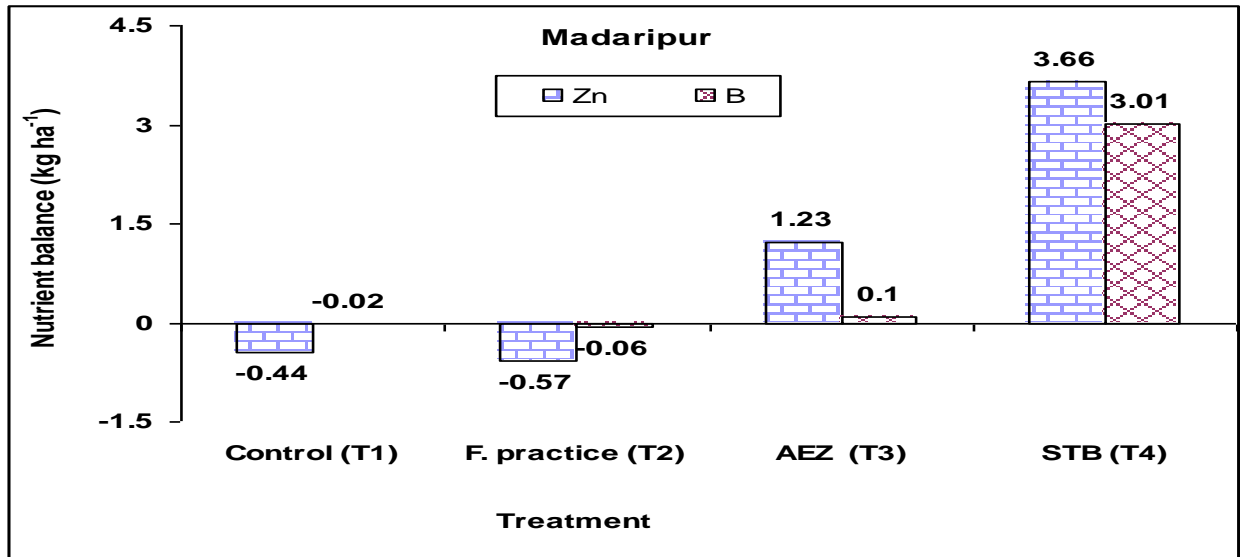

Fig. 4. Apparent nutrient balance of zinc and boron under Wheat-Mungbean - $T$. aman cropping pattern at Madaripur.

\section{Soil fertility}

Initial soil samples were collected from the experimental field and post harvest soil samples were also collected from each treated plot after two cycles of Wheat-Mungbean-T. aman cropping pattern for analyzing different soil properties viz. soil $\mathrm{pH}$, organic matter, total $\mathrm{N}$ and available $\mathrm{P}, \mathrm{K}, \mathrm{S}, \mathrm{Ca}, \mathrm{Mg}, \mathrm{Zn}$ and $\mathrm{B}$. The initial and post harvest soil results are presented in Table 8. Initially the soil $\mathrm{pH}$ was 7.3, but after completion of two crop cycles and incorporation of mungbean stover and other crop residues in soil, the $\mathrm{pH}$ remained unchanged although minor variation existed. A minor change in soil fertility occurred from initial status due to different fertilizer management practices over two years. Soil 
test based fertilizer application $\left(\mathrm{T}_{4}\right)$ tended to maintain the initial fertility or increased slightly (Table 8). Aggarwal et al. (1997) observed that the fertility of soil was improved due to incorporation of green manure into soil. The treatment $\mathrm{T}_{4}$ showed an encouraging effect on organic matter, $\mathrm{N}, \mathrm{P}, \mathrm{S}, \mathrm{Ca}, \mathrm{Zn}$ and $\mathrm{B}$ only. Potasium $(\mathrm{K})$ and $\mathrm{Mg}$ slightly decreased in all plots over the initial status. The available $\mathrm{Zn}$ and $\mathrm{B}$ content of the soil slightly decreased when they were not applied, but remained almost static or increase when applied (Table 8).

Table 8. Initial and post soil fertility status after two cycles of Wheat-Mungbean-T. aman cropping pattern due to different fertilizer management practices

\begin{tabular}{|c|c|c|c|c|c|c|c|c|c|c|}
\hline \multirow[b]{2}{*}{ Treatment } & \multirow[b]{2}{*}{$\mathrm{pH}$} & \multirow{2}{*}{$\begin{array}{c}\mathrm{OM} \\
(\%)\end{array}$} & \multirow{2}{*}{$\begin{array}{c}\text { Total } \\
\mathrm{N} \\
(\%) \\
\end{array}$} & $\mathrm{Ca}$ & $\mathrm{Mg}$ & $\mathrm{K}$ & $\mathrm{P}$ & $\mathrm{S}$ & $\mathrm{Zn}$ & $\mathrm{B}$ \\
\hline & & & & \multicolumn{3}{|c|}{ Meq. $100 \mathrm{~g}^{-1}$} & \multicolumn{4}{|c|}{$\mu \mathrm{g} \mathrm{g}^{-1}$} \\
\hline Initial & 7.3 & 1.32 & 0.063 & 10.3 & 3.10 & 0.14 & 13.5 & 18.0 & 1.20 & 0.14 \\
\hline $\operatorname{Control}\left(\mathrm{T}_{1}\right)$ & 7.3 & 1.31 & 0.062 & 10.0 & 2.99 & 0.11 & 13.8 & 17.6 & 1.17 & 0.12 \\
\hline F. practice $\left(T_{2}\right)$ & 7.3 & 1.35 & 0.064 & 10.3 & 3.00 & 0.12 & 14.3 & 18.0 & 1.17 & 0.12 \\
\hline $\operatorname{AEZ}\left(T_{3}\right)$ & 7.2 & 1.38 & 0.065 & 10.3 & 3.02 & 0.12 & 14.3 & 18.3 & 1.26 & 0.14 \\
\hline $\operatorname{STB}\left(\mathrm{T}_{4}\right)$ & 7.2 & 1.40 & 0.068 & 10.4 & 3.02 & 0.13 & 14.5 & 19.0 & 1.34 & 0.16 \\
\hline
\end{tabular}

\section{Economic analysis}

Gross returns varied in different treatments Wheat-Mungbean-T. aman cropping system which were directly related to the price that received from the product. The gross returns were highest (Tk. $263260 \mathrm{ha}^{-1} \mathrm{yr}^{-1}$ ) in the treatment $\mathrm{T}_{4}$ followed by $T_{3}$ and $T_{2}$ and the lowest was in control treatment (Table 8). Cost of cultivation was involved with wage rate, pesticides, irrigation and fertilizers cost. Data on cost and return analysis showed that the maximum gross margin (Tk. $173663 \mathrm{ha}^{-1} \mathrm{yr}^{-1}$ ) was calculated from $\mathrm{T}_{4}$ and minimum from $\mathrm{T}_{1}$. The gross margin by $\mathrm{T}_{4}$ was increased two fold over control $\left(\mathrm{T}_{1}\right)$ treatment due to get higher crop yield. Similar observation was showed by Malika et al. (2015). The highest benefit cost ratio (3.29) was obtained from $\mathrm{T}_{3}$ followed by $\mathrm{T}_{4}(2.94)$ and $\mathrm{T}_{2}$ (2.88). Considering the benefit cost ratio (BCR) $\mathrm{T}_{3}$ treatment showed ranked first followed by $\mathrm{T}_{4}$. However, the cost of production of $\mathrm{T}_{3}\left(\mathrm{Tk} .72881 \mathrm{ha}^{-1} \mathrm{yr}^{-1}\right)$ was lower than $\mathrm{T}_{4}\left(\mathrm{Tk} .89595 \mathrm{ha}^{-1} \mathrm{yr}^{-1}\right)$.

Table 9. Economic analysis of Wheat-Mungbean-T.aman cropping pattern affected by different nutrient managements (after completing two years cycle)

\begin{tabular}{l|c|c|c|c}
\hline \multirow{2}{*}{ Treatment } & Variable cost & Gross return & Gross margin & BCR \\
\cline { 2 - 5 } & \multicolumn{4}{|c}{ Tk. ha $\mathbf{~} \mathbf{y r}^{-1}$} \\
\hline Control $\left(\mathrm{T}_{1}\right)$ & 59875 & 163699 & 103824 & 2.73 \\
F. practice $\left(\mathrm{T}_{2}\right)$ & 71549 & 206148 & 134599 & 2.88 \\
AEZ $\left(\mathrm{T}_{3}\right)$ & 72881 & 240062 & 167181 & 3.29 \\
STB $\left(\mathrm{T}_{4}\right)$ & 89595 & 263260 & 173663 & 2.94 \\
\hline
\end{tabular}


Input prices: Urea=Tk. $12 \mathrm{~kg}^{-1}$, T.S.P $=$ Tk. $22 \mathrm{~kg}^{-1}, \mathrm{MoP}=\mathrm{Tk} .20 \mathrm{~kg}^{-1}$, Gypsum $=$ Tk.6 kg-1 , Zinc sulphate $=$ Tk. $120 \mathrm{~kg}^{-1}$, Boric acid $=\mathrm{Tk} .300 \mathrm{~kg}^{-1}$, Rovral fungicide $=$ Tk.250 $100^{-\mathrm{g}}$, Bavistin fungicide $=$ Tk.200 100 ${ }^{-\mathrm{g}}$, Provex fungicide $=$ Tk.3200 $\mathrm{kg}^{-1}$, Ripcord insecticide $=$ Tk.105 $100^{-\mathrm{g}}$, Karate insecticide $=$ Tk.450 $500^{-\mathrm{ml}}$, Plowing $=$ Tk.1400 ha ${ }^{-1}$ (one pass), Labour wage $=$ Tk.125 day $^{-1}$, Wheat seed $=$ Tk.25 kg ${ }^{-1}$, Mungbean seed $=$ Tk.60 kg ${ }^{-1}$, T. aman rice seed $=T k .35 \mathrm{~kg}^{-1}$.

Output prices: Wheat $=T \mathrm{Tk} .18 .75 \mathrm{~kg}^{-1}$, Mungbean $=\mathrm{Tk} .55 \mathrm{~kg}^{-1}$, T. aman rice $=$ Tk.19 $\mathrm{kg}^{-1}$, Wheat straw rate $=$ Tk. $1 \mathrm{~kg}^{-1}$, Rice straw $=$ Tk. $1.25 \mathrm{~kg}^{-1}$.

The BCR and gross margin was the highest in $\mathrm{T}_{3}$ and $\mathrm{T}_{4}$, respectively and economically viable. The fertilizer doses under $\mathrm{T}_{3}$ were very low, hence its nutrients balance was highly negative after control. On the other hand, the fertility of soil remains almost static or increased slightly due to $\mathrm{T}_{4}$ treatment. Therefore, considering the gross margin and soil fertlity the treatment $\mathrm{T}_{4}$ is preferable to $\mathrm{T}_{3}$.

\section{Conclusion}

The magnitude of negative balance was greater with $\mathrm{N}$ and $\mathrm{K}$ followed by $\mathrm{Mg}$ and $\mathrm{Ca}$. Nitrogen and $\mathrm{K}$ mining occur remarkably from the soil. So, the rates of application of these two nutrients should be increased. Considering the gross margin and soil fertility the soil test based fertilizer management practice (STB) is economically profitable and sustainable. Therefore, Wheat-Mungbean-T. aman cropping pattern is good in sustaining soil fertility and found economically sound and viable for Madaripur region in Bangladesh.

\section{References}

Aggarwal P., D. K. Parashar, V. Kumar, R. P. Gupta. 1997. Effect of kharif green manuring and rabi tillage on physical properties of clay loam under rice-wheat rotation. J. Indian Soc. Soil Sci. 45(3): 434-438.

Alam, M. S., N. Islam, M. Jahiruddin. 2000. Effect of zinc and boron application on the performance of local and hybrid maize. Bangladesh J. Soil Sci. 26: 95-101.

Ali, A. M., M. R. Alam, M. S. H. Molla, F. Islam. 2010. Crop productivity as affected by fertilizer management options in Boro-T.aman cropping pattern at farmers fields. Bangladesh J. Agril. Res. 35(2), 287-296.

Bijay, S. and S. Yadvinder. 2002. Potassium nutrition of Rice-Wheat cropping system. Department of soil Science, Punjab Agricultural University, Ludhiana, India.

Biswas, M. A., A. Anwar, R. I. Mondal, M. A. Hossain, R. Uddin. 2009. Integrated Nutrient Management for Sustaining Soil Fertility and Yield of Lentil-MungbeanT.Aman cropping pattern at Jessore Region. Annual Research Report, Pulses Research Centre, BARI, Gagipur, Pp 116-119. 
FRG. 2012. Fertilizer Recommendation Guide. Published by Bangladesh Agricultural Research Council Dhaka, Bangladesh.

Ghosh, B. K. 2011. Determinants of the Changes in Cropping Pattern in India: 1970-71 to 2006-07. Bangladesh Dev. Stud. Vol. XXXIV, No. 2.

Hanks, R. J. and G. L. Ashcroft. 1980. Applied Soil Physics; Soil Water and Temperature Applications. Springer-verlag Berlin, Heidelberg, Newyork. Pp. 1-159.

Hossain, MA, Jahiruddin M, Islam MR, Mian MH. 2008. The requirement of zinc for improvement of crop yield and mineral nutrition in the maize-mungbean-rice system. Plant Soil. 306: 13-22.

Islam M. R., M. R. Karim, T. M. Riasat, M. Jahiruddin. 1996. Growth and yield of BR11 rice under different levels of sulphur, zinc and boron fertility at two locations of Bangladesh. Thai J. Agril Sci. 29: 37-42.

Jahan, M. A. H. S., M. A. R. Sarkar, N. C. D. Barma, M. N. A. Mondal, M. N. S. Ferdousi. 2015a. Grain yield, nutrient balance and economics of $\mathrm{T}$. aman rice cultivation as influenced by nutrients management. Bangladesh J. Agril. Res. 40(1): 17-34.

Jensen and L. Thomas. 2010. Soil pH and the availability of plant nutrients.IPNI Plant Nutrition Today, No. 2, www. ipni.net

Katoh M, Iwata A, Shaku I, Nakajima Y, Matsuya K, Kimura M. 2003. Impact of water percolation on nutrient leaching from an irrigated paddy field in Japan. Soil Use and Management, 19, 298-304.

Kumar S. and M. Singh (Editors). 2009. 25 Years of Pulses Research at IIPR, 1984-2009, Published by: Indian Institute of Pulses Research, Kanpur 208024, India. Pp.68-69.

Malika, M., M. R. Islam, M. R. Karim, A. Huda, M. Jahiruddin. 2015. Organic and inorganic fertilizers influence the nutrient use efficiency and yield of a rice variety BINA dhan7. Acad. Res. J. Agril. Sci. Res. 3(7): 192-200. ISSN: 2360-7874.

Rahman, M. H., M. R. Islam, M. Jahiruddin, M. Q. Haque. 2011. Economics of fertilizer use in the Maize-Mungbean/Dhaincha-T.aman rice cropping pattern. J. Bangladesh Agril. Univ. 9(1), 37-42.

Saleque, M. A., J. Timsina, G. M. Panaullah, M. Ishaque, A. B. M. B. U. Pathan, D. J. Connor, P. K. Saha, M. A. Quayyum, E. Humphreys, C. A. Meisner. 2006. Nutrient Uptake and Apparent Balances for Rice-Wheat Sequences. II. Phosphorus. J. Plant Nutr. 28: $157-172$.

Srinivasarao, C. H., B. K. Ramachandrappa, V. S. Jakkula, S. Kundu, B. Venkateswarlu, A. L. Pharande, V. R. Manideep, R. P. Naik, K. Venkanna. 2014. Nutrient Balance after Thirteen Years of Organic and Chemical Nutrient Management and Yield Sustainability of Groundnut-Fingermillet Rotation in Rainfed Alfisols of Semi-Arid India. J. Indian Soc. Soil Sci. 62(3): 235-247.

Tarafder, M. A., M. Q. Haque, M. M. Rahman, M. R. Khan. 2008. Direct and residual effect of sulphur and zinc on potato-boro-T. aman rice cropping pattern. Progress Agric. 19(1): 33-38. 
Timsina, J., L. J. Mangi, K. Majumdar. 2010. Rice-maize systems of South Asia: current status, future prospects and research priorities for nutrient management. Plant Soil. 335: 65-82.

Ved Ram, M. Ali, K. U. Mishra, R. M. Upadhyay. 2008. Studies on sulphur, zinc and biofertilizers on yield, yield attributes and nutrient content at different growth stages in mungbean. Journal of Food Legumes. 21(4): 240-242.

Yu, YL, LH Xue and L. Z. Yang,. 2014. Winter legumes in rice crop rotations reduces nitrogen loss, and improves rice yield and soil nitrogen supply. Agron. Sustain Dev. 34, 633-40. 